\title{
Academy of Medicine-Ministry of Health Clinical Practice Guidelines: Attention Deficit Hyperactivity Disorder
}

\author{
Daniel SS Fung, Choon Guan Lim, John Chee Meng Wong, Koon Hock Ng, Christopher Cheng Soon Cheok, \\ Jennifer Sie Hee Kiing, Shang Chee Chong, June Lou, Mary Lourdes Daniel, Desmond Ong, Charity Low, \\ Sharifah Mariam Aljunied, Pui Meng Choi, Kala Mehrotra, Carolyn Kee, Ivy Leung, Lee Chen Yen, Geraldine Wong, \\ Poh Yin Lee, Bella Chin, Hwee Chien $\underline{\mathrm{Ng}}$
}

\begin{abstract}
The Academy of Medicine (AMS) and the Ministry of Health (MOH) have developed the clinical practice guidelines on Attention Deficit Hyperactivity Disorder (ADHD) to provide doctors and patients in Singapore with evidencebased treatment for ADHD. This article reproduces the introduction and executive summary (with recommendations from the guidelines) from the $\mathrm{MOH}$ clinical practice guidelines on ADHD, for the information of SMJ readers. Chapters and page numbers mentioned in the reproduced extract refer to the full text of the guidelines, which are available from the Ministry of Health website: http://www.moh.gov.sg/content/moh_web/healthprofessionalsportal/doctors/guidelines/ cpg_medical.html.The recommendations should be used with reference to the full text of the guidelines. Following this article are multiple choice questions based on the full text of the guidelines.
\end{abstract}

\subsection{Background information}

Attention deficit hyperactivity disorder (ADHD) is a neuro-developmental disorder, characterised by the presence of early-onset persistent, pervasive and impairing hyperactiveimpulsive and/or inattentive symptoms. The worldwide pooled prevalence was about $5.29 \%$. While there has been no epidemiological study on the prevalence of this condition among children in Singapore, a local study reported the prevalence of externalising problems to be $4.9 \%$ among Singaporean primary school children. While ADHD symptoms decline with age, a meta-analysis reported that a significant proportion might have residual symptoms in adulthood. ADHD has been reported to be associated with negative outcomes, including poor academic achievement, reduced self-esteem and higher smoking rate. This condition is often picked up when the child is young, and children with ADHD are often treated by child psychiatrists and paediatricians.

\subsection{Objectives and scope of guideline}

These guidelines are not meant to be viewed as a protocol, but rather, to provide a framework to:

- Approach the assessment process, with the goals of arriving at a diagnosis and planning treatment.

- Inform the practitioner about the level of evidence for medication available in Singapore, to aid the decision about medication use.

- Inform about the evidence for non-pharmacological treatment approaches to help professionals plan treatment and discuss these with parents.

\subsection{Target group}

The target group of these guidelines are professionals who come into contact with children and adolescents below the age of 18 who have attention deficit hyperactivity disorder (ADHD). The information is intended to help professionals plan the treatment for such children and adolescents. Professionals should exercise caution when extrapolating the evidence beyond this stated age group.

\subsection{Guideline development}

These guidelines were produced by a multi-disciplinary workgroup appointed by the Academy of Medicine, Singapore. The workgroup comprised psychiatrists, paediatricians, educational psychologists, a medical social worker, a pharmacist, an advanced practice nurse and a parent representative.

\subsection{Review of guidelines}

Evidence-based clinical practice guidelines are only as current as the evidence that supports them. Users must keep in mind that new evidence could supersede recommendations in these guidelines. The workgroup advises that these guidelines be scheduled for review five years after publication, or earlier if new evidence appears that requires substantive changes to the recommendations.

\section{EXECUTIVE SUMMARY OF RECOMMENDATIONS}

Details of the recommendations listed can be found in the main text as the pages indicated. 


\section{Definition and diagnostic classification}

B A diagnosis of attention deficit hyperactivity disorder should be made through a thorough clinical assessment, which should include an interview with the parent or significant caregiver of the child (pg 12).

Grade B, Level $2^{++}$

B When diagnosing attention deficit hyperactivity disorder, in addition to information from interviews with parents or caregivers, information from another adult who has interacted with the child in another setting (e.g. school teachers) should also be obtained (pg 12).

Grade B, Level $2^{++}$

B Before diagnosing attention deficit hyperactivity disorder (ADHD), a careful evaluation to exclude psychiatric or medical conditions which can account for ADHD-like symptoms should be performed (pg 13).

Grade B, Level 2++

B Attention deficit hyperactivity disorder is a diagnosis that should be considered when a pre-schooler presents with disruptive behaviour (pg 13).

Grade B, Level $2^{++}$

C The clinician should assess a child diagnosed with attention deficit hyperactivity disorder for co-morbid conditions (pg 13).

Grade C, Level $2^{+}$

C If there is a suspected learning disorder, appropriate psycho-educational or speech and language assessments should be sought from the appropriate specialists (pg 14).

Grade C, Level $2^{+}$

A There is no need for investigations such as thyroid function test, lead level or brain imaging to be done when assessing a child for attention deficit hyperactivity disorder, unless there is another medical indication (pg 14).

Grade A, Level $1^{+}$

A Electroencephalogram is not recommended as a diagnostic tool for attention deficit hyperactivity disorder in clinical practice (pg 15).

Grade A, Level 1+

\section{Overview of treatment for ADHD}

D Clinicians who treat adolescents with attention deficit hyperactivity disorder should plan for the transition to adult health services in advance, discuss this with the patients and their families, and ensure that they can continue to receive care (pg 17).

Grade D, Level 4

\section{Psychosocial/alternative/complementary interventions}

B After diagnosis, doctors should provide appropriate education about attention deficit hyperactivity disorder to children, families and teachers (pg 18).

Grade B, Level 1+

A Doctors should consider educating parents of children with attention deficit hyperactivity disorder about behaviour management strategies, or refer them to professionals who can do so [e.g. psychologists] (pg 19).

Grade A, Level $1^{++}$

B Parent training should be offered for parents of pre-school children with attention deficit hyperactivity disorder (pg 20).

Grade B, Level 1+

B Doctors should consider referring parents of children and adolescents with attention deficit hyperactivity disorder for parent training programmes offered within the community, particularly when negative parenting practices are identified (pg 20).

Grade B, Level 1+

D During the delivery of parent training, the professional should consider the use of behaviour management strategies which are more likely to be acceptable to the parents, based on an understanding of their cultural background (pg 21).

Grade D, Level 3

GPP Family therapy may be considered for the family of a child or adolescent with attention deficit hyperactivity disorder if severe disruption in relationships within the family is evident (pg 21).

C

Academic interventions should be considered for the child with attention deficit hyperactivity disorder and should be made in consultation with educational professionals who work closely with the child in the learning or school context. [Guidelines for communications between the physician and the child's school are provided in Annex 5.] (pg 22).

Grade C, Level 2+

GPP Parents and caregivers should be encouraged to actively share information about the child's attention deficit hyperactivity disorder condition with his school, and collaborate with professionals and teachers in preparing the child for the educational setting (pg 24).

GPP

B Social skills training alone is not recommended for the management of attention deficit hyperactivity disorder (pg 25).

Grade B, Level 1+ 
B Cognitive-behavioural therapy alone is not recommended for the management of attention deficit hyperactivity disorder (pg 25).

Grade B, Level 1+

B There is no clear evidence for food additives and sugars to be related to attention deficit hyperactivity disorder. Parents and children should be advised to control food items containing additives or high sugar content that have been observed to consistently provoke physical or behavioural reactions (pg 26).

Grade B, Level 1+

C A restrictive elimination diet is not recommended for the management of attention deficit hyperactivity disorder (pg 27).

Grade C, Level 2++

B Omega-3 fatty acid supplementation may be used as an adjunctive treatment for attention deficit hyperactivity disorder (pg 27).

Grade B, Level 1+

B Neurofeedback should not be used alone for the treatment of attention deficit hyperactivity disorder (pg 29).

Grade B, Level 1+

A Cognitive remediation alone is not recommended for the treatment of attention deficit hyperactivity disorder with significant impairment (pg 29).

Grade A, Level $1^{+}$

D A referral to an Occupational Therapist may be considered for children with sensory processing or motor skill deficits in addition to attention deficit hyperactivity disorder (pg 30).

Grade D, Level 3

\section{Pharmacological treatment}

A When medication is considered for the treatment of attention deficit hyperactivity disorder, methylphenidate should be considered first (pg 32).

Grade A, Level 1+

B Methylphenidate may be used for long term treatment of attention deficit hyperactivity disorder symptoms, although the benefits of treatment should be reviewed regularly (pg 32).

Grade B, Level 1+

B Drug holidays during treatment with methylphenidate may be considered in order to limit adverse effects. Attention deficit hyperactivity disorder symptoms and impairment during the non-medication days should be monitored (pg 33).

Grade B, Level 1+

A

The height, weight and body-mass-index (BMI) of children receiving treatment with methylphenidate should be regularly monitored (pg 33).

Grade A, Level 1++

The height, weight and body-mass-index (BMI) of children receiving treatment with methylphenidate should be monitored every 6 months. If there is concern about slowing of growth rate, the need for continued medication use should be reviewed and jointly decided with parents, and there may be a need to evaluate for other medical reasons explaining this (pg 34).

Grade D, Level 4

B During treatment with methylphenidate, start at a low dose and slowly titrate upwards according to the child's response, or adjust the timing of medication, to minimise short-term adverse effects (pg 34).

Grade B, Level 1+

A careful personal and family history of cardiovascular disease should be taken before starting medication treatment for attention deficit hyperactivity disorder. Children with pre-existing cardiac problems should be referred to a cardiologist for evaluation before treatment with methylphenidate or atomoxetine is initiated (pg 34).

Grade C, Level $2^{+}$

A Methylphenidate may be used to treat attention deficit hyperactivity disorder in children with comorbid tic disorder but treatment should be stopped if the tics worsen following treatment (pg 35).

Grade A, Level 1+

B

The use of methylphenidate should be considered when treating attention deficit hyperactivity disorder in the presence of co-morbid disruptive behavioural disorder (pg 35).

Grade B, Level $1^{+}$

B

The use of an extended-release methylphenidate instead of immediate-release methylphenidate should be considered if there is concern about medication abuse. Medication use by these patients should be carefully monitored (pg 36).

Grade B, Level 1+

Methylphenidate may be considered for the treatment of attention deficit hyperactivity disorder in individuals who have also been diagnosed with autistic spectrum disorder. Care should be taken to watch for side effects (pg 36).

Grade A, Level $1^{+}$

Atomoxetine may be used for the treatment of attention deficit hyperactivity disorder symptoms when there is 
increased risk with methylphenidate use [e.g. high risk of abuse or diversion] (pg 37).

Grade A, Level 1+ should not be used for the treatment of attention deficit hyperactivity disorder symptoms (pg 41).

Grade C, Level 2+

A To improve treatment adherence, treatment should be individualised for each patient with attention deficit hyperactivity disorder, and the parents' and their child's preferences should be considered (pg 41).

Grade A, Level $1^{+}$ use should be reviewed and jointly decided with parents, and there may be a need to evaluate for other medical reasons explaining this (pg 37).

Grade A, Level $1^{++}$

D The height, weight and body-mass-index (BMI) of children receiving treatment with atomoxetine should be monitored every 6 months (pg 38).

Grade D, Level 4

\section{Cost-effectiveness issues}

A Although medication is a cost-effective treatment for attention deficit hyperactivity disorder (ADHD), treatment for ADHD should be individualised and other factors (e.g. presence of co-morbidity) should be considered before initiating medications (pg 42).

Grade A, Level $1^{++}$

C The combination of methylphenidate and atomoxetine schoolers should be considered only if psychosocial interventions have failed. Care should be taken to regularly assess response and monitor for side effects, so as to decide if medication should continue to be administered (pg 41).

Grade A, Level $1^{++}$

B Atomoxetine may be used as first line treatment when there is comorbid attention deficit hyperactivity disorder and tic disorder (pg 38).

Grade B, Level 1+ 


\section{SINGAPORE MEDICAL COUNCIL CATEGORY 3B CME PROGRAMME (Code SMJ 201408C)}

These questions are based on the full text of the guidelines which may be found at http://www.moh.gov.sg/content/moh_web/ healthprofessionalsportal/doctors/guidelines/cpg_medical.html

Question 1. ADHD can be diagnosed when:

(a) The child meets the symptom checklist as required by DSM or ICD.

(b) Both parents and teachers rate the child with high scores on a standard rating scale.

(c) A thorough clinical history from parents and teachers or other caregivers fulfills all DSM or ICD criteria.

(d) A child has been observed to exhibit symptoms in a clinic setting.

Question 2. Recognised risks associated with methylphenidate use include:
(a) Reduced appetite.
(b) Reduced rate of physical growth.
(c) Increased cardiovascular risk.
(d) Risk of medication abuse or diversion.

Question 3. Features present in all cases of ADHD include:

(a) Symptoms present before the age of 7 .

(b) Significant hyperactive and/or impulsive symptoms.

(c) Significant impairment from the symptoms.

(d) Presence of symptoms in more than one setting.

Question 4. Regarding treatment for ADHD:

(a) Methylphenidate should be used when ADHD is of at least moderate severity.

(b) Parent training should be offered when a pre-schooler is diagnosed with ADHD.

(c) Behaviour treatment is useful when there is significant comorbid anxiety symptom.

(d) Methylphenidate should be considered when there is comorbid oppositional defiant disorder or conduct disorder.

Question 5. If a parent is not keen to have the child receive medication for the treatment of ADHD, the following will be reasonable next strategies:

(a) Educate about behavioural management strategies.

(b) Refer for neurofeedback therapy.

(c) Suggest parent communicate the diagnosis to the school teachers and seek their help.

(d) Educate teachers about classroom management strategies, at least in writing.
True False

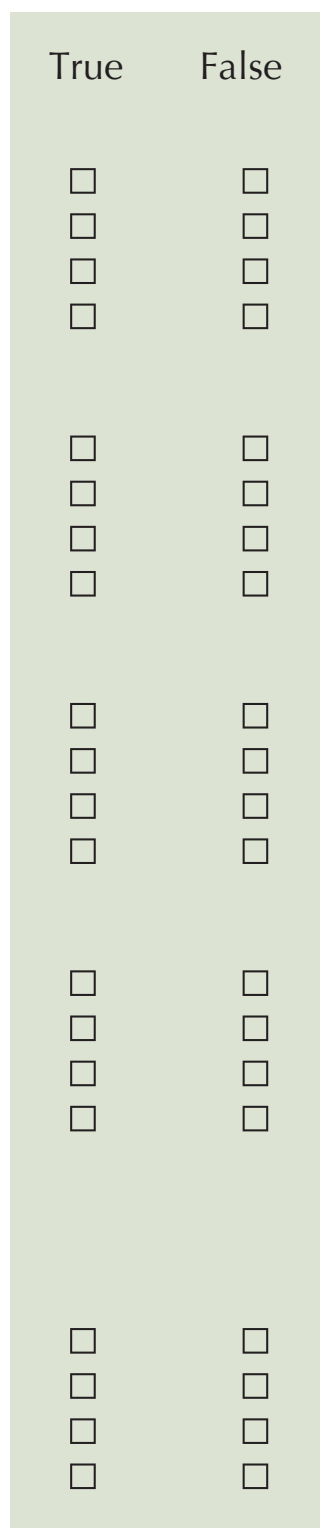

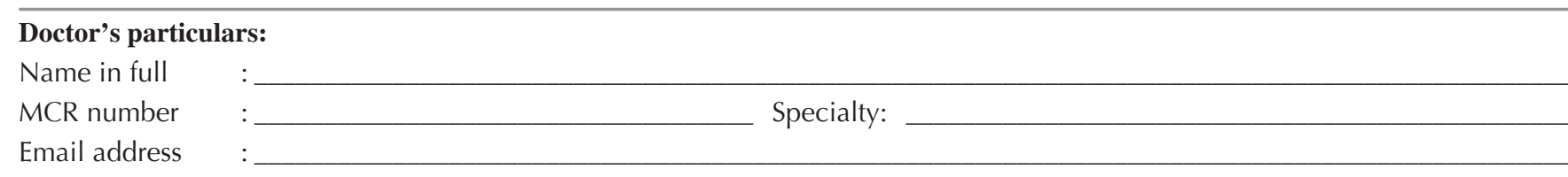

\section{SUBMISSION INSTRUCTIONS:}

(1) Log on at the SMJ website: http://www.sma.org.sg/publications/smjcurrentissue.aspx and select the appropriate set of questions. (2) Provide your name, email address and MCR number. (3) Select your answers and click "Submit".

\section{RESULTS:}

(1) Answers will be published in the SMJ October 2014 issue. (2) The MCR numbers of successful candidates will be posted online at the SMJ website by 30 September 2014. (3) Passing mark is $60 \%$. No mark will be deducted for incorrect answers. (4) The SMJ editorial office will submit the list of successful candidates to the Singapore Medical Council. (5) One CME point is awarded for successful candidates.

Deadline for submission: (August 2014 SMJ 3B CME programme): 12 noon, 24 September 2014. 